\title{
Prevalence of Malocclusion in Students in a Subpopulation of Mato Grosso, Brazil
}

\author{
${ }^{1}$ Alessandra N Porto, ${ }^{2}$ Andreza MF Aranha, ${ }^{3}$ Alexandre M Borba, ${ }^{4}$ Evanice VM Menezes, ${ }^{5}$ Cieila C Machado \\ ${ }^{6}$ Mariane M Dias, ${ }^{7}$ Orlando A Guedes, ${ }^{8}$ Matheus C Bandéca, ${ }^{9}$ Álvaro H Borges, ${ }^{10}$ Luiz ER Volpato
}

\section{ABSTRACT}

Aim: To determine the prevalence of malocclusion in children and students enrolled in public schools and in the Araguaia Family Health Strategy in the municipality of Guarantã do Norte, Mato Grosso, Brazil.

Materials and methods: A cross-sectional study was conducted with randomly selected children and adolescents from 6 to 16 years. The students were examined at the selected institutions under natural light by a trained examiner. The sample size was calculated using a $95 \%$ confidence interval and data were analyzed by descriptive statistics using absolute and relative frequencies.

Results: A total of 400 children and adolescents were selected, $176(44 \%)$ males and 224 females (56\%). At facial analysis, the most prevalent facial patterns were mesofacial (43\%), dolichofacial (41\%), and brachyfacial (16\%). In facial profile, $48 \%$ were convex, $39 \%$ straight, and $13 \%$ concave. The occlusal relationship found was $44 \%$ class I, $36 \%$ class II, and $20 \%$ class III; $62 \%$ vertical malocclusions, $54 \%$ horizontal and $66 \%$ harmful habits (bucconasal breathing, finger sucking, and pacifier sucking) were also found.

Conclusion: The prevalence of malocclusion was high; over $50 \%$ had some type of occlusal changes.

Clinical significance: Cross-sectional studies have their importance, as they show the disease situation in different places, subsiding decision-making for prevention and treatment strategies, adapting to the regional financial reality in an attempt to reduce health inequalities among the various social and economic strata of the population.

Keywords: Malocclusion, Prevalence, Students.

How to cite this article: Porto AN, Aranha AMF, Borba AM, Menezes EVM, Machado CC, Dias MM, Guedes OA, Bandéca MC, Borges AH, Volpato LER. Prevalence of Malocclusion in Students in a Subpopulation of Mato Grosso, Brazil. World J Dent 2018;9(4):260-264.

Source of support: Nil

Conflict of interest: None

1-4,7,9,10Department of Postgraduate Program, School of Dentistry, University of Cuiabá, Cuiabá, Mato Grosso, Brazil

${ }^{5,6}$ Department of Postgraduate Program, Syndicate of Dentistry Cuiabá, Mato Grosso, Brazil

${ }^{8}$ Department of Postgraduate Program in Dentistry, CEUMA University, São Luis, Maranhão, Brazil

Corresponding Author: Alessandra N Porto, Av. Beira Rio 3100, Cuiabá, Mato Grosso, Brazil, e-mail: aleporto@terra. com.br

\section{INTRODUCTION}

Malocclusion, defined as growth and development disarrangements that affect the occlusion of teeth, is considered a public health problem because of its high prevalence and possibility of negatively impacting quality of life. ${ }^{1}$ It affects the function, appearance, impair social interaction, self-esteem, and the psychological well-being of affected individuals. ${ }^{1,2}$

Malocclusions have a multifactorial origin which may be caused by general factors, such as congenital and genetic factors, nutritional deficiencies, or abnormal pressure habits; or by local factors, located directly in the arcade, as supernumerary teeth, dental caries, and premature loss of deciduous teeth. ${ }^{3}$

The association between malocclusions and changes in the speech articulation and swallowing functions has received greater emphasis, especially in the association with mouth breathing, due to the severity of the associated alterations, such as obstructive sleep apnea and postural changes. ${ }^{4}$ It is considered the third oral problem in the oral diseases priority scale, after dental caries and periodontal disease, according to the World Health Organization. ${ }^{5}$

In Brazil, the National Oral Health Survey conducted in $2010^{6}$ found the presence of at least one dental malocclusion condition in $66.7 \%$ of the 5 -year-old children, $38.9 \%$ of the 12 -year-olds, and $34.9 \%$ in the age group of 15 to 19 years. Most epidemiologic studies show data of the urban population of large and medium-sized cities, especially those of easy access.

The municipality of Guarantã do Norte is located $729 \mathrm{~km}$ from Cuiabá, capital of Mato Grosso state, in the Mid-West Brazil. It has a population of 31,642 inhabitants, spread over $4,735 \mathrm{~km}^{2}$, and its economic base is mineral extraction. The identification of the oral health status of populations is fundamental for planning and evaluating health promotion actions. ${ }^{6,7}$

This study aimed to determine the prevalence of malocclusions of schoolchildren aged from 6 to 16 years of both genders in the Amazon Region.

\section{MATERIALS AND METHODS}

This quantitative, exploratory cross-sectional study was conducted in the municipality of Guarantã do Norte, 
Mato Grosso state, Brazil. The population of the study was children and adolescents of both genders aged 6 to 16 years selected by convenience sampling. Data collection was carried out between April and May 2012, at the Darcy Ribeiro Municipal School and the Family Health Strategy unit.

The study protocol was approved by the Ethics Committee in Research of the University of Cuiabá (Protocol \#026/2012). All legal guardians were informed about the research and its protocol and signed the informed consent term before the children underwent the medical history assessment and clinical examination.

The exclusion criteria were individuals with prior orthodontic treatment or under orthodontic treatment; individuals with premature loss of first permanent molars or permanent molars with partially or completely destroyed crowns; agenesis or unerupted permanent molars. Data were collected by a calibrated single examiner.

The clinical examination and the analysis of dental arches were performed under direct vision and good lighting, with the aid of disposable wooden spatula (Theoto S/A Ind e Com., Jundiaí, SP, Brazil); dental floss; and pencils, millimeter rulers, and specially designed files. In this study, the evaluation of malocclusions was performed using Angle's classification ${ }^{8}$ criteria, supplemented by data on the vertical interincisal trespass, horizontal interincisal trespass, and posterior transversal relationship.

The history record and clinical examination files were composed of: (1) Patient identification; (2) facial analysis: facial symmetry with facial pattern (mesofacial, dolichofacial, and brachyfacial) and profile facial view (straight, concave, and convex); (3) occlusal relationship (molar relationship between the first upper and lower molars according to Angle's classification-classes I, II,

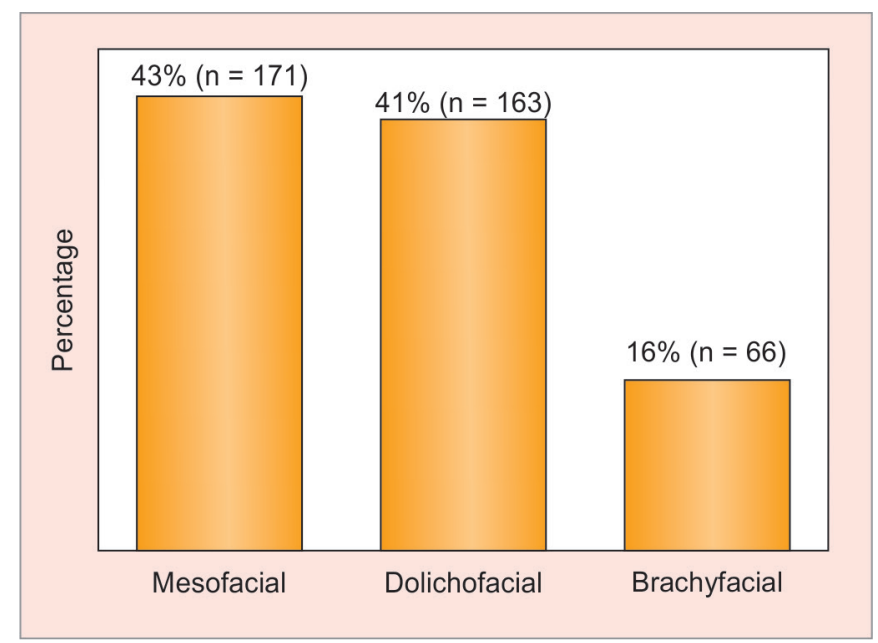

Graph 1: Distribution of the facial patterns in the studied population and III); (4) posterior transversal relationship (unilateral or bilateral crossbite); (5) vertical anterior relationship (open bite and deep overbite); (6) deleterious oral habits (finger or pacifier sucking and mouth breathing). Data were analyzed using descriptive statistics with the use of tables and graphs containing absolute and relative frequencies.

\section{RESULTS}

From the total of 400 students aged 6 to 16 years assessed, 224 (56\%) were girls and 176 (44\%) were boys. A high prevalence of malocclusion was found among the evaluated individuals. The facial analysis showed that the most frequent pattern group was mesofacial $(43 \%, n=171)$, followed by dolichofacial $(41 \%, n=163)$ and brachyfacial $(16 \%, n=66)$ (Graph 1).

The most frequent facial profile group was the convex $(48 \%, \mathrm{n}=194)$, followed by straight $(39 \%, \mathrm{n}=155)$ and concave $(13 \%, \mathrm{n}=51)$ (Graph 2). Evaluating the occlusal relationship, it was found that $44 \%(\mathrm{n}=176)$ were class I, 36\% $(n=144)$ were class II, and $20 \%(n=80)$ were class III (Graph 3).

The vertical interincisal trespass showed a normal relationship in 38\% $(n=152)$, open bite in $43 \%(n=172)$, and deep overbite in 19\% $(n=76)(G r a p h 4)$. The transverse relationship showed a normal relationship in $46 \%$ ( $n=184)$, posterior bilateral crossbite in $28 \%(n=113)$, posterior unilateral crossbite in $8 \%$ (33), anterior crossbite in $14 \%(n=57)$, and anterior posterior crossbite in 4\% (13) (Graph 5).

Regarding the presence of deleterious oral habits, it was found that they were absent in 34\% ( $n=136), 14 \%$ ( $n=56)$ had finger sucking habits, $11 \%(n=44)$ had pacifier sucking habits, and 41\% ( $\mathrm{n}=164)$ had mouth breathing habits (Graph 6).

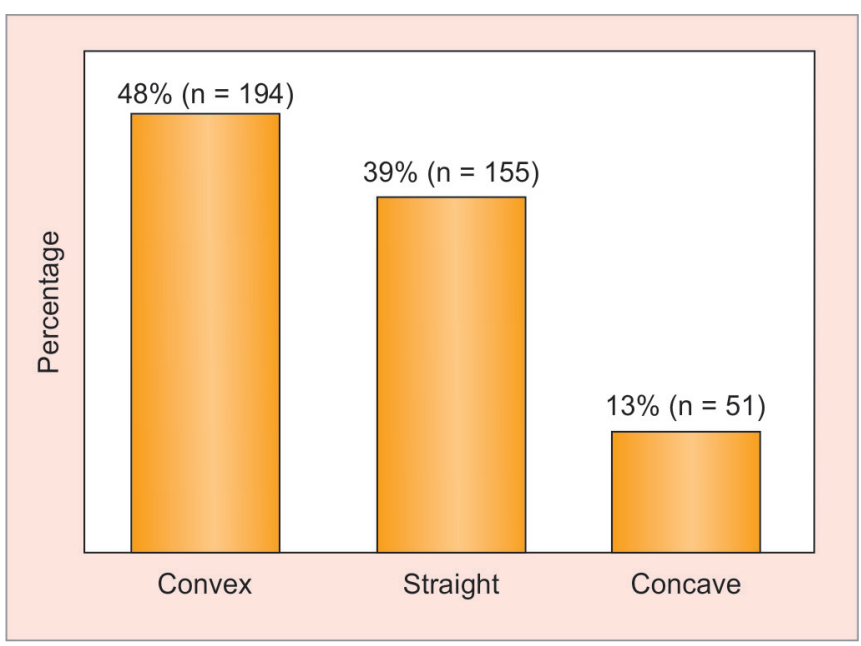

Graph 2: Distribution of the facial profiles in the studied population 


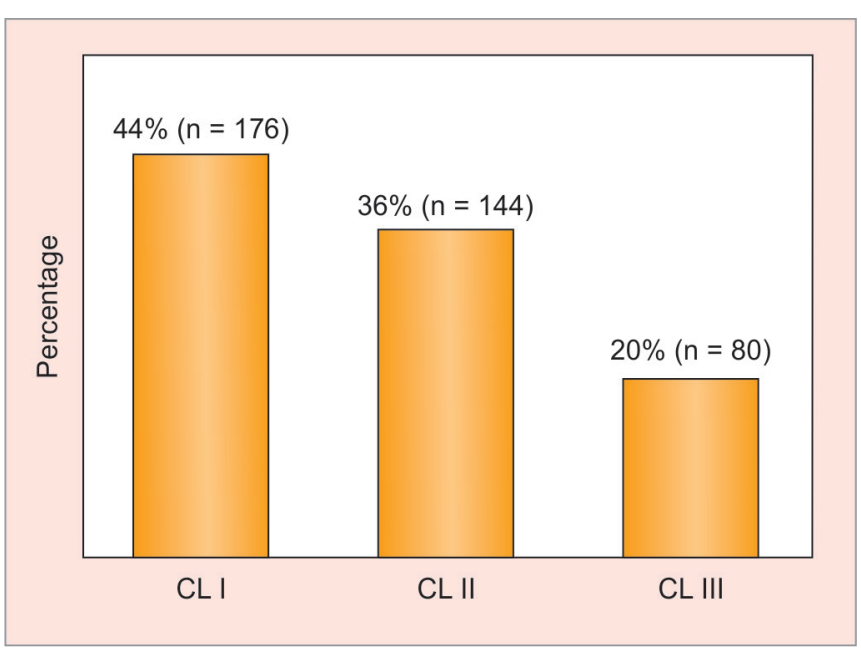

Graph 3: Distribution of malocclusion type according to Angle's classification

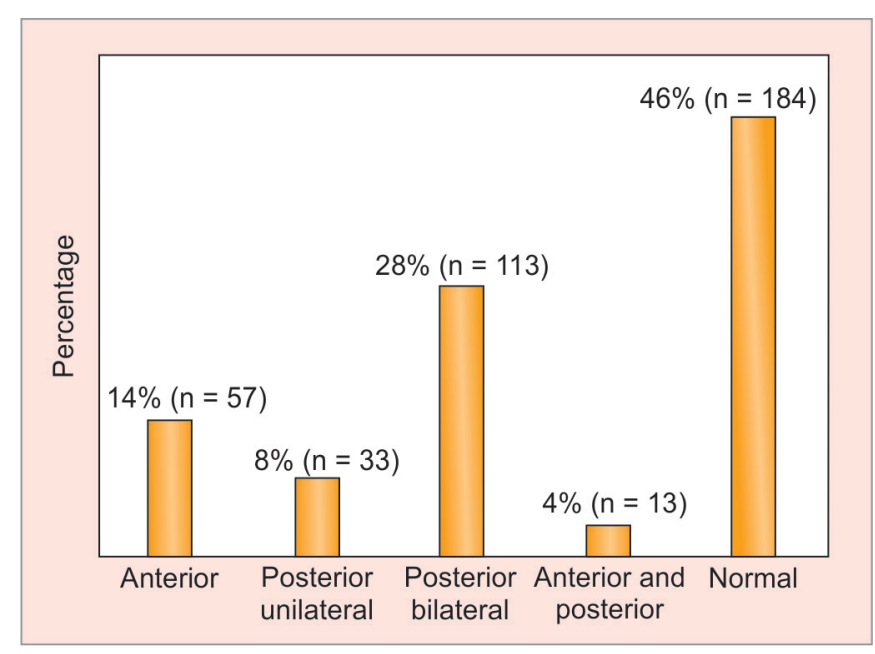

Graph 5: Distribution of the presence and type of crossbite

Further, it was found that $74 \%$ of the examined individuals had at least one permanent or deciduous tooth absent, $15 \%$ were deciduous, and $59 \%$ permanent teeth.

\section{DISCUSSION}

Malocclusion is a public health problem in the contemporary world and, thus, widely studied over the years. ${ }^{3}$ In Brazil, most studies on the prevalence of malocclusion in the deciduous dentition are performed in schoolchildren. ${ }^{9,10}$

In this study, malocclusion was closely related to the poor dental conditions of the study population: Dental caries, premature loss of deciduous and permanent teeth, and oral habits in all evaluated racial groups (whites, blacks, and Asians), with high prevalence, similar to other studies in Brazil. ${ }^{3,11}$

The high prevalence of malocclusion in this study could be influenced by the socioeconomic status of the population, since it consisted of public health care and

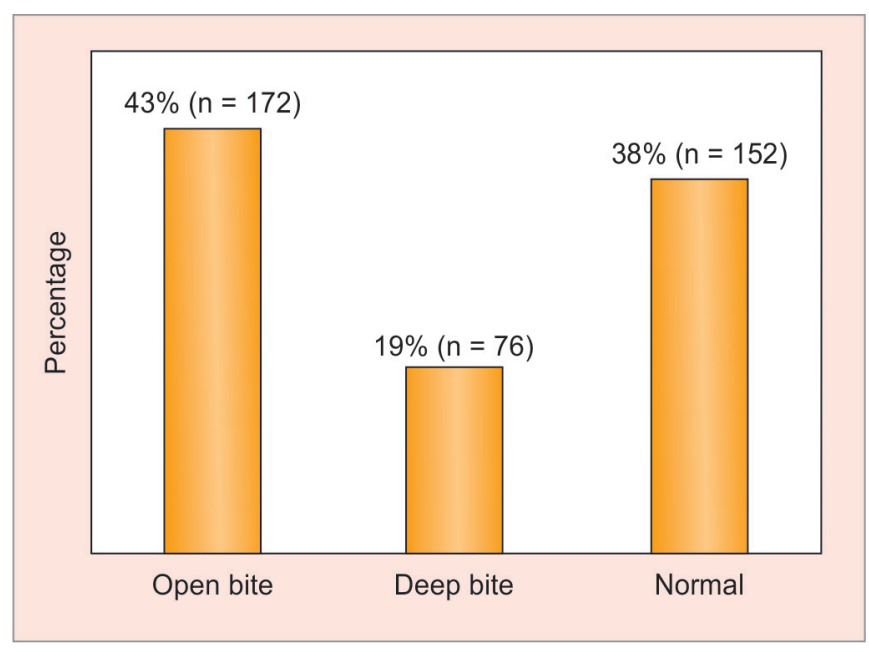

Graph 4: Distribution of overbite in the studied population

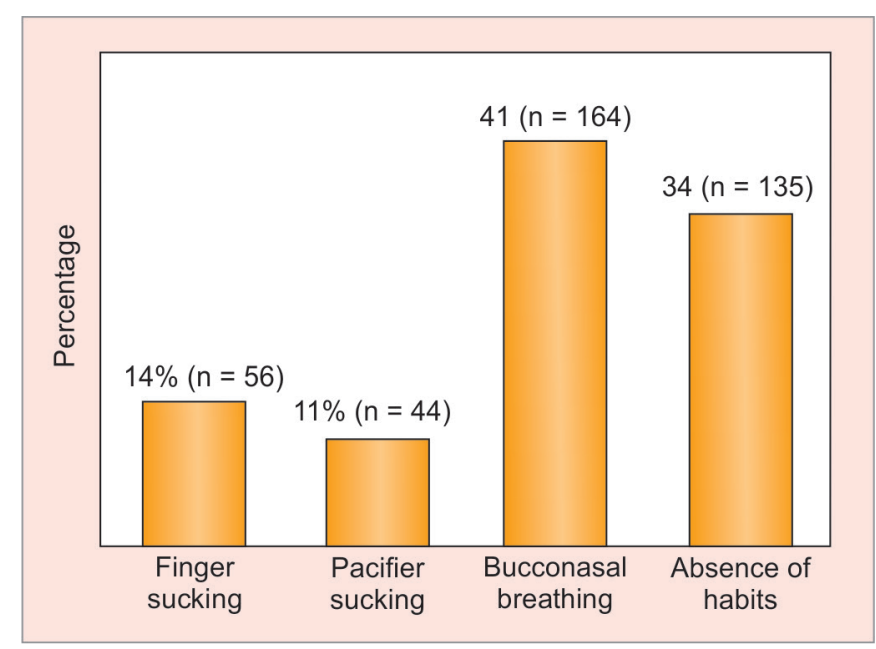

Graph 6: Distribution of the presence of harmful habits and neurovegetative function

education services users. Positive and negative associations between malocclusion and socioeconomic characteristics were found, although conclusive answers on the subject are scarce. ${ }^{12,13}$

According to Angle, ${ }^{8}$ the classification is based on where the buccal groove of the mandibular first molar contacts the mesiobuccal cusp of the maxillary first molar on the cusp (class I, neutrocclusion, or normal occlusion); distal to the cusp by at least the width of a premolar (class II, distocclusion); or mesial to the cusp (class III, mesiocclusion). Thus, complementing is necessary to enable a sustainable treatment plan, based on the five main characteristics of malocclusion: facial profile, alignment, and vertical, transverse, and sagittal deviations. ${ }^{14}$

As for occlusal relationship, class I presented with the highest prevalence, followed by classes II and III, similar to a previous study also conducted in Brazi ${ }^{15}$ and different from other studies in which class II presented with higher prevalence. ${ }^{16}$ 
The high prevalence of malocclusion found in this study may be due to the high tooth loss encountered, which is a known factor for exacerbating problems of space, rotations, and others. According Melsen and Terp, ${ }^{17}$ individuals with tooth loss are nearly eight times more likely to develop malocclusion when compared with individuals who did not lose teeth.

The most prevalent facial pattern was mesofacial, followed by dolichofacial and brachyfacial, and facial profiles were convex, followed by the straight and concave, similar to some studies that have shown the same facial pattern, emphasizing that the dolichofacial pattern is related to a higher prevalence of anterior open bite and posterior crossbite. $^{18}$

Over $50 \%$ of the 400 students assessed had at least one type of malocclusion, whether of vertical or transverse origin. Considering the presence of vertical problems $(62 \%)$, the most frequent was the open bite $(43 \%)$, followed by normal (38\%) and then deep bite (19\%), as found in some studies ${ }^{10,19}$ and different from others. ${ }^{15}$

The posterior crossbite bilateral $(28 \%)$ and posterior unilateral $(8 \%)$ were more prevalent than the anterior crossbite (14\%), agreeing with Souki et $\mathrm{al}^{20}$ and disagreeing with Bittencourt and Machado. ${ }^{15}$

The high frequency of anterior open bite and posterior crossbite in the present study may be related to deleterious oral habits, such as mouth breathing and finger and/or pacifier sucking interfering in the formation and development of the orofacial complex during childhood and adolescence similar to the study of Dimberg et al. ${ }^{21}$ It is known that if those habits are interrupted before the ages of 3 to 4 , the malocclusion may undergo a spontaneous correction process in most cases. ${ }^{21}$

According to Peres et $\mathrm{al}_{1}{ }^{22}$ some risk factors increase the chances of developing malocclusions, as precocious loss of deciduous teeth [odds ratio (OR) of 6,408], loss of permanent teeth $(\mathrm{OR}=7,856)$, mouth-nose breathing $(\mathrm{OR}=6,408)$, pacifier use $(\mathrm{OR}=3,432)$, and finger sucking $(\mathrm{OR}=1,684)$, highlighting the need of early orthodontic intervention.

This study presented the weaknesses inherent of cross-sectional studies, as it did not establish causal relationships to the malocclusions; it did not evaluate the variable time and its contextual influence on individual condition. But cross-sectional studies have their importance, as they show the disease situation in different places, subsiding decision-making for prevention and treatment strategies, adapting to the regional financial reality in an attempt to reduce health inequalities among the various social and economic strata of the population.

\section{CONCLUSION}

The findings conclude that the prevalence of malocclusion was high; over $50 \%$ had some type of occlusal changes.

\section{ACKNOWLEDGMENT}

The authors would like to thank the Master Program in Integrated Dentistry Science, University of Cuiabá, UNIC.

\section{REFERENCES}

1. MarquesLS, BarbosaCC,Ramos-JorgeML,PordeusIA,PaivaSM. Malocclusion prevalence and orthodontic treatment need in 10-14-year-old schoolchildren in Belo Horizonte, Minas Gerais State, Brazil: a psychosocial focus. Cad Saude Publica 2005 Jul-Aug;21(4):1099-1106.

2. Choi SH, Kim BI, Cha JY, Hwang CJ. Impact $f$ malocclusion and common oral diseases on oral health-related quality of life in young adults. Am J Orthod Dentofacial Orthop 2015 May;147(5):587-595.

3. de Almeida MR, Pereira AL, de Almeida RR, de AlmeidaPedrin RR, Filho OG. Prevalence of malocclusion in children aged 7 to 12 years. Dental Press J Orthod 2011 Jul-Aug;16(4): 123-131.

4. Altalibi M, Saltaji H, Roduta Roberts M, Major MP, MacLean J, Major PW. Developing an index for the orthodontic treatment need in paediatric patients with obstructive sleep apnoea: a protocol for a novel communication tool between physicians and orthodontists. BMJ Open 2014;4:e005680.

5. Word Health Organization. Health through oral health: guidelines for planning and monitoring for oral health care. World Health Organization and Federation Dentaire Internationale. London: Quintessence; 1989.

6. Ministry of Health. Oral Health Coordination. Smiling Brazil program manual. Brazil: Ministry of Health; 2010. Available from: http://portal.saude.gov.br/portal/saude/area. cfm?id_area $=406$.

7. Oliveira AG, Unfer B, Costa IC, Arcieri RM, Guimarães LO, Saliba NA. Epidemiological study in oral health: analysis of the methodology proposed by World Health Organization. Rev Bras Epidemiol 1998 Aug;1(2):5-10.

8. Angle EH. Classification of malocclusion. Dent Cosmos 1899 Mar;41:248-264.

9. de Sousa RV, Ribeiro GL, Firmino RT, Martins CC, GranvilleGarcia AF, Paiva SM. Prevalence and associated factors for the development of anterior open bite and posterior crossbite in the primary dentition. Braz Dent J 2014;25(4):336-342.

10. Fernandes KP, Amaral MT. Frequency of malocclusions among 3-6-year-old schoolchildren in the city of Niteroi, RJ, Brazil. Pesq Bras Odontoped Clin Integr 2008 MayAug;8(2):147-151.

11. Antunes JL, Pegoretti T, de Andrade FP, Junqueira SR, Frazão P, Narvai PC. Ethnic disparities in the prevalence of dental caries and restorative dental treatment in Brazilian children. Int Dent J 2003 Feb;53(1):7-12.

12. Tickle M, Kay EJ, Bearn D. Socio-economic status and orthodontic treatment need. Community Dent Oral Epidemiol 1999 Dec;27(6):413-418.

13. Joury E, Johal A, Marcenes W. The role of socio-economic position in predicting orthodontic treatment outcome at the end of 1 year of active treatment. Eur J Orthod 2011 Jun;33(3): 263-269.

14. Ackerman JL, Proffit WR. The characteristics of malocclusion: a modern approach to classification and diagnosis. Am J Orthod 1969 Nov;56(5):443-454. 
15. Bittencourt MA, Machado AW. An overview of the prevalence of malocclusion in 6 to 10-year-old children in Brazil. Dental Press J Orthod 2010 Nov-Dec;15(6):113-122.

16. Uslu O, Akcam MO, Evirgen S, Cebeci I. Prevalence of dental anomalies in various malocclusions. Am J Orthod Dentofacial Orthop 2009 Mar;135(3):328-335.

17. Melsen B, Terp S. The influence of extractions caries cause on the development of malocclusion and need for orthodontic treatment. Swed Dent J Suppl 1982;15:163-169.

18. Nogueira Fialho MP, Pinzan-Vercelino CR, Nogueira RP, Gurgel JA. Relationship between facial morphology, anterior open bite and non-nutritive sucking habits during the primary dentition stage. Dental Press J Orthod 2014 MayJun;19(3):108-113.
19. Dimberg L, Lennartsson B, Arnrup K, Bondemark L. Prevalence and change of malocclusions from primary to early permanent dentition: a longitudinal study. Angle Orthod 2015Sep; 85(5):728-734.

20. Souki BQ, Pimenta GB, Souki MQ, Franco LP, Becker HM, Pinto JA. Prevalence of malocclusion among mouth breathing children: do expectations meet reality? Int J Pediatr Otorhinolaryngol 2009 May;73(5):767-773.

21. Dimberg L, Bondemark L, Söderfeldt B, Lennartsson B. Prevalence of malocclusion traits and sucking habits among 3-year-old children. Swed J Dent 2010 Jan;34(1):35-42.

22. Peres KG, Frazão P, Roncalli AG. Epidemiological pattern of severe malocclusions in Brazilian adolescents. Rev Saude Publica 2013 Dec;47(Suppl 3):109-117. 